\title{
Highly effective fat suppression in clinical T1- weighted imaging of ischemic and non-ischemic heart disease with DeSPAIR
}

\author{
Wolfgang G Rehwald ${ }^{1,2^{*}}$, Christoph J Jensen², Elizabeth Jenista² ${ }^{2}$ Stephen Darty ${ }^{2}$, Deneen Spatz ${ }^{2}$, Raymond J Kim² \\ From 15th Annual SCMR Scientific Sessions \\ Orlando, FL, USA. 2-5 February 2012
}

\begin{abstract}
Summary
Complete fat suppression is highly desirable for inversion recovery (IR) imaging of both ischemic and nonischemic heart disease. It allows for improved visualization of scar without confounding fat signal, and for assessment of fatty infiltrations, which both appear bright on T1-weighted contrast-enhanced images. For example, arrhythmogenic right ventricular dysplasia (ARVD) is characterized by fibrofatty replacement of the right ventricular (RV) myocardium. Screening tests are critical as patients may be asymptomatic, but at increased risk for sudden cardiac death. Such tests must differentiate myocardial fibrosis from fatty infiltrations, as fatty infiltrations without RV fibrosis have been observed in healthy subjects. Standard fat saturation works poorly in clinical protocols, prompting us to design a double Spectral Selection Attenuated Inversion Recovery (double SPAIR, DeSPAIR) module that completely nulls fat signal in IR sequences. In 22 patients with ischemic and non-ischemic heart disease, we demonstrate excellent performance of this technique compared to standard fat saturation.
\end{abstract}

\section{Background}

Protocols that impart T1-weighting by an IR pulse, such as delayed enhancement, can incorporate a standard fat saturation pulse to suppress fat, but perform poorly with clinically useful readout lengths. Alternatively, sequences without an IR pulse can null fat by a fatselective inversion using SPAIR. Unfortunately, this approach cannot be readily applied to sequences with an IR for T1-weighting, since fat would experience the

'Siemens Healthcare, Chapel Hill, NC, USA

Full list of author information is available at the end of the article
IR pulse and would not be fully recovered when the SPAIR pulse is applied, resulting in such a short effective inversion time (TI_fat) that fat nulling is impossible. We developed a method to overcome this problem by combining a double SPAIR (DeSPAIR) module with an IR pulse to suppress fat while maintaining T1-weighting.

\section{Methods}

The DeSPAIR module integrated into an IR sequence is shown in figure 1a. SPAIR \#1 immediately follows the non-selective IR (NSIR) pulse to re-invert fat magnetization and keep it at $+\mathrm{M} 0$. To null fat, SPAIR $\# 2$ follows at time TI_fat prior to readout (RO) of the k-space center. Normal myocardium is nulled by the NSIR placed TI_myo prior to the k-space center. Dark myocardium, dark fat and bright scar result in the image. We acquired 3 images per patient in 22 patients $(9$ at $1.5 \mathrm{~T}$, 13 at $3 \mathrm{~T}, 4$ ischemic, 18 non-ischemic) using no fat suppression (NONE), fat saturation (FS), and DeSPAIR. In each image, we measured signal-to-noise (SNR) in multiple red dashed fat regions of interest (ROI) (figure 1b). To quantify suppression efficiency, SNR of FS and DeSPAIR in each ROI was normalized to SNR of NONE in the same ROI and expressed as relative SNR in percent. An ANOVA with Bonferroni correction was applied to test for statistical differences between groups NONE, FS, and DeSPAIR. Cavity and normal myocardium SNR were measured to evaluate if FS or SPAIR would affect SNR.

\section{Results}

Typical delayed enhancement images in an ARVD patient using NONE, FS, and DeSPAIR at 3T are shown in figure 1b. Visual inspection showed excellent suppression of fatty infiltration in the RV by DeSPAIR (3 white 
a)

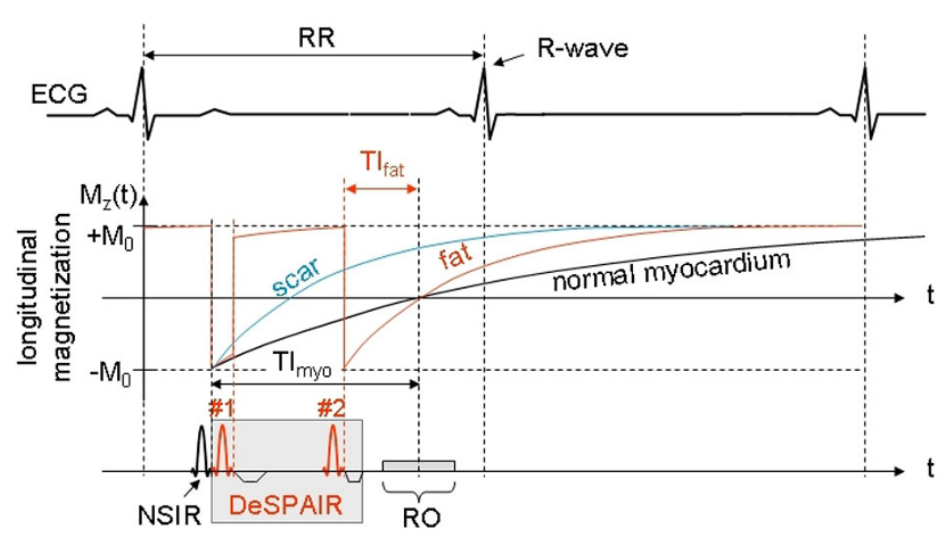

b)

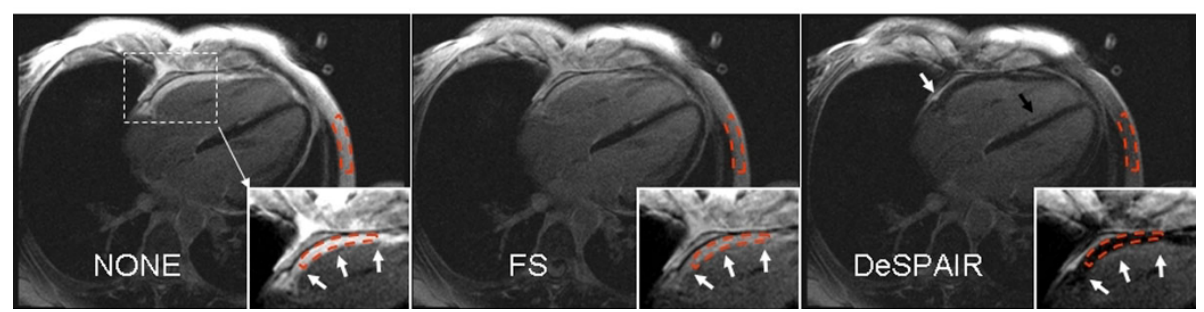

Figure 1 a) shows the DeSPAIR module integrated into a segmented IR gradient echo sequence and the resulting T1 recovery curves. NSIR and SPAIR pulses are timed to null myocardium and fat at the k-space center. Typical parameters at 1.5T (MAGNETOM Avanto, Siemens Healthcare) and 3 T (Verio) were, respectively: Tl adjusted to null normal myocardium (300-400 ms), trigger pulse 2, fov $360 \times 270 \mathrm{~mm}$, matrix $256 \times 125$, segments 21, flip angle $25^{\circ}\left(15^{\circ}\right.$ ), receiver bandwidth 130 (399) Hz/pixel, TE 3.85 (1.66) ms, TR 8.9 (4.4) ms, slice thickness 7 (6) mm. 1b) shows delayed enhancement images at $3 \mathrm{~T}$ in an ARVD patient without fat suppression, FS, and DeSPAIR. Fat suppression was poor by FS, but excellent by DeSPAIR. Note the fatty infiltration of the RV freewall (3 white arrows), pericardial effusion (white arrow) only seen in the DeSPAIR image, and septal fibrosis (black arrow).

Table 1 Relative Fat SNR, Cavity and Myocardial SNR

\begin{tabular}{ccccccc}
\hline field strength & \multicolumn{1}{c}{$1.5 T$} & & & \\
\hline sup-pression method & NONE & FS & DeSPAIR & NONE & FS \\
\hline relative fat SNR & $100 \%$ & $101.63 \% \pm 29.92 \% \#$ & $39.52 \% \pm 19.87 \% *$ & $100 \% *$ & $68.79 \% \pm 28.88 \% *$ & $32.72 \% \pm 15.53 \% *$ \\
\hline cavity SNR & $19.75 \pm 10.29$ & $25.13 \pm 12.41 \#$ & $16.91 \pm 9.28 \#$ & $49.82 \pm 37.16$ & $49.26 \pm 32.29 \#$ & $44.92 \pm 32.95 \#$ \\
\hline myocardial SNR & $3.78 \pm 2.40$ & $5.59 \pm 3.28 \#$ & $3.85 \pm 2.05 \#$ & $7.46 \pm 5.36$ & $6.79 \pm 4.44 \#$ \\
\hline
\end{tabular}

shown: mean \pm stdev $^{*}$ : statistically different from NONE $(p<0.001)$ \#: statistically identical to NONE $(p>0.05)$.

arrows), and hardly any by FS. Septal hyperenhancement was present with and without fat suppression, indicating fibrosis (black arrow). Statistical analysis of relative SNR revealed significantly suppressed fat by DeSPAIR at $1.5 \mathrm{~T}$ and $3 \mathrm{~T}$ (see table 1). Fat was not suppressed by FS at $1.5 \mathrm{~T}$ and significantly suppressed at $3 \mathrm{~T}$, but less than by DeSPAIR (table 1). Neither cavity nor myocardial SNR were statistically different $(\mathrm{p}>0.05)$ between any of the techniques, at both field strengths.

\section{Conclusions}

DeSPAIR reliably nulled fat signal at both field strengths for clinically relevant RO lengths. DeSPAIR performed much better than FS at both field strengths, but the difference was particularly striking at $1.5 \mathrm{~T}$. DeSPAIR required no manual parameter adjustment and no post processing - only a simple change to an existing sequence. In clinical practice, DeSPAIR can help establish the diagnosis of ARVD even in early stages, and potentially improve patient outcome.

\section{Funding}

NIH grant 5ROIHL064726-07.

\section{Author details}

'Siemens Healthcare, Chapel Hill, NC, USA. ²Duke Cardiovascular MR Center, Duke University Medical Center, Durham, NC, USA.

Published: 1 February 2012 
doi:10.1186/1532-429X-14-S1-053

Cite this article as: Rehwald et al:: Highly effective fat suppression in

clinical T1-weighted imaging of ischemic and non-ischemic heart

disease with DeSPAIR. Journal of Cardiovascular Magnetic Resonance 2012

14(Suppl 1):053.

Submit your next manuscript to BioMed Central and take full advantage of:

- Convenient online submission

- Thorough peer review

- No space constraints or color figure charges

- Immediate publication on acceptance

- Inclusion in PubMed, CAS, Scopus and Google Scholar

- Research which is freely available for redistribution

Submit your manuscript at 